\title{
Nonlocal Fractional Semilinear Delay Differential Equations in Separable Banach spaces
}

\author{
V.Dhanapalan ${ }^{1}$, M.Thamilselvan ${ }^{2}$, M.Chandrasekaran ${ }^{3}$ \\ ${ }^{I}$ (Department of Mathematics, Government College of Technology, Coimbatore -641013.Tamilnadu, India.) \\ ${ }_{2}^{2}$ (Department of Physics, Thanthai Periyar Government Institute of Technology, Vellore-632002.Tamilnadu, \\ India.) \\ ${ }^{3}$ (Department of Mathematics, Higher College of Technology, Muscat, Sultanate of Oman.)
}

\begin{abstract}
In this article, we study the existence of mild solutions for fractional semilinear differential equations with nonlocal conditions in separable Banach spaces. The result is obtained. The result is obtained using the Hausdorff measure of noncompactness and the Schauder fixed point theorem .
\end{abstract}

Keywords: Fractional differential equation, nonlocal conditions, Housdorff measure of noncompactness, mild solution.

\section{INTRODUCTION}

Let $X$ be a separable Banach space endowed with norm $\|\|, A:. D(A) \varphi \subset X \rightarrow X$ the infinitesimal generator of a strongly continuous semigroup of bounded linear operators $\{T(t)\}_{t \geq 0}, D(A)$ the domain of $A$. We consider the nonlocal fractional semilinear differential equation ${ }^{\mathrm{c}} D_{t}^{\alpha} u(t)=A u(t)+f(t, u(\sigma(t))), t \in[0, b]$,

$(1.1)$

$u(0)=g(u)$,

where $0<\alpha<1,{ }^{\mathrm{C}} D_{t}^{\alpha}$ is the $\alpha$-order Caputo fractional derivative operator, $f, g$, and $\sigma$ are functions to be specified later.

Recently, the theory of fractional differential equations has attracted much interest due to their many applications in physics, chemistry, biology, finance and so on. We refer to the books of Podlubny [17], Samko et al [18], Kilbas et al [9] and the papers of Nigmatullin [14], Orisingher and Beghin [16], Meerschaert et al [13], Hahn et al [7], Li et al [10].

The semilinear evolution nonlocal Cauchy problem was initiated by Byszewski [4].The nonlocal condition can be applied in physics with better effect in applications than the classical initial condition since nonlocal conditions are usually more precise for physical measurements than the classical initial condition. Lin and Liu [11] studied semilinear integrodifferential equations with nonlocal Cauchy problems under Lipchitz type conditions. Ntouyas and Tsamatos [15] studied the global existence of solutions semilinear evolution equations with nonlocal conditions via a fixed point analysis approach. Fu and Ezzinbi [6] studied the existence of mild and strong solutions of semilinear neutral functional differential evolution equations with nonlocal conditions by using fractional power of operators and Sadovskii's fixed point theorem. Xue [19] studied the existence of mild solutions for semilinear differential equations with nonlocal initial conditions in separable Banach spaces. Xue [20] discussed the semilinear nonlocal differential equations when the semigroup $T(t)$ generated by the coefficient operator is compact and the nonlocal term $g$ is not compact.Fan and $\mathrm{Li}$ [5] discussed the existence for impulsive semilinear differential equations with nonlocal conditions by using Sadovskii's fixed point theorem and Schauder's fixed point theorem.

In this article, we shall study the existence of mild solutions of (1.1) by using the Hausdorff measure of compactness and fixed point theorems. We assume that the semigroup $T(t)$ generated by 
the coefficient operator is equicontinuous. The compactness of $T(t)$ of $f$ and the Lipchitz condition of $f$ are the special cases our conditions. Therefore, the results in this paper generalize and improve some of previous ones in this field.

The article is organized as follows. Section 2 contains some preliminaries about fractional calculus and the Hausdorff's measure of compactness. In Section 3 the existence result is given.

\section{PRELIMINARIES}

Let $(X,\|\cdot\|)$ be a separable Banach space Banach space and $\mathbb{R}_{+}=[0, \infty)$. we denote by $C([0, b] ; X)$ the space of $X$-valued continuous functions on $[0, \mathrm{~b}]$ with the norm

$$
\|u\|_{C}:=\sup _{t \in[0, b]}\|u(t)\|
$$

and by $L^{1}([0, b] ; X)$, we denote the space of $X$-valued Bochner integrable functions $u:[0, b] \rightarrow X$ with the norm $\|u\|_{L^{1}([0, b] ; X)}=\int_{0}^{b}\|u(t)\| d t$.

\subsection{Definition}

The Riemann- Liouville fractional integral of $u:[0, b] \rightarrow X$ of order $\alpha \in(0, \infty)$ is defined by

$$
J_{t}^{\alpha} u(t)=\frac{1}{\Gamma(\alpha)} \int_{0}^{t}(t-s)^{\alpha-1} u(s) d s .
$$

The Riemann-Liouville fractional derivative of $u:[0, b] \rightarrow X$ of order $\alpha \in(0,1)$ is defined by

$$
D_{t}^{\alpha} u(t)=\frac{1}{\Gamma(1-\alpha)} \frac{d}{d t} \int_{0}^{t}(t-s)^{-\alpha} u(s) d s .
$$

The Caputo fractional derivative of $u:[0, b] \rightarrow X$ of order $\alpha \in(0,1)$ is defined by

$$
{ }^{\mathrm{c}} D_{t}^{\alpha} u(t)=D_{t}^{\alpha}(u(t)-u(0))
$$

We recall the Hausdorff measure of noncompactness $\beta_{Y}(\cdot)$ defined on a bounded subset of $B$ of Banach space $Y$ by $\beta_{Y}(B)=\inf \{\varepsilon>0 ; B$ has a finite $\varepsilon$ - net in $Y\}$.

Some basic properties of $\beta_{Y}(\cdot)$ are presented in the following lemma.

\subsection{Lemma ([1]).}

Let $Y$ be real Banach space and $B, C \subseteq Y$ be bounded, the following properties are satisfied:

(1) $\mathrm{B}$ is precompact if and only if $\beta_{Y}(B)=0$;

(2) $\beta_{Y}(B)=\beta_{Y}(\bar{B})=\beta_{Y}(\operatorname{conv} B)$, where $\bar{B}$ and $\operatorname{conv} B$ mean the closure and convex hull of $B$ respectively;

(3) $\beta_{Y}(B) \leq \beta_{Y}(C)$ and $B \subseteq C$;

(4) $\beta_{Y}(B+C) \leq \beta_{Y}(B)+\beta_{Y}(C)$ where $B+C=\{x+y$; $x \in B, y \in C\}$;

(5) $\beta_{Y}(B \cup C) \leq \max \left\{\beta_{Y}(B), \beta_{Y}(C)\right\}$;

(6) $\beta_{Y}(\lambda B)=|\lambda| \beta_{Y}(B)$ for any $\lambda \in R$;

(7) if the mapping $Q: D(Q) \subseteq Y \rightarrow Z$ is Lipchitz continuous with constant $k$, then $\beta_{Z}(Q B) \leq k \beta_{Y}(B)$ for any bounded subset $B \subseteq D(Q)$, where $Z$ is a Banach space;

(8) If $\left\{W_{n}\right\}_{n=1}^{\infty}$ is a decreasing of bounded closed nonempty subsets of $Y$ and $\lim _{n \rightarrow \infty} \beta_{Y}\left(W_{n}\right)=0$, then $\cap_{n=1}^{\infty} W_{n}$ is nonempty and compact in $Y$. The map $Q: W \subseteq Y \rightarrow Y$ is said to be a $\beta_{Y}$-contraction if there exists appositive constant $k<1$ such that $\beta_{Z}(Q(C)) \leq k \beta_{Y}(C)$ for any bounded closed subset $C \subseteq W$ where $Y$ is a Banach space.

\subsection{Lemma (Darbo-Sadovskii).}


If $W \subseteq Y$ is bounded closed and convex, the continuous map $Q: W \rightarrow W$ is a $\beta_{Y}$-contraction, then the map $\mathrm{Q}$ has at least one fixed point in $\mathrm{W}$.

In this article, without loss of generality, we denote the Hausdorff measure of noncompactness of $X$ and $C([0, b] ; X)$.

\subsection{Lemma $([1])$.}

If $W \subset C([0, b] ; X)$ is bounded and equicontinuous, then the set $\beta(W(t))$ is continuous on $[0, \mathrm{~b}]$ and

$$
\beta(W)=\sup _{t \in[0, b]} \beta(W(t)) \text {. }
$$

\subsection{Lemma ([3]).}

If $\left\{u_{n}\right\}_{n=1}^{\infty} \subset L^{1}([0, b] ; X)$ satisfies $\left|u_{n}(t)\right| \leq \varphi(t)$ a.e. on [0,b] for all $n \geq 1$ with some $\varphi \in L^{1}\left([0, b] ; \mathbb{R}_{+}\right)$, then

$$
\beta\left(\left\{\cup_{n=1}^{\infty} \int_{0}^{t} u_{n}(s) d s\right\}\right) \leq \int_{0}^{t} \beta\left(\left\{\cup_{n=1}^{\infty} u_{n}(s)\right\}\right) d s .
$$

\subsection{Definition}

A $C_{0}$ semigroup $T(t)$ is said to be equicontinuous if the mapping $t \rightarrow\{T(t) x: X \in B\}$ is equicontinuous at $t>0$ for all bounded set $B$ in Banach space $X$.

\subsection{Definition ([17]).}

The Mainardi's function is defined by

$$
M_{\alpha}(z)=\sum_{n=0}^{\infty} \frac{(-z)^{n}}{n ! \Gamma(-\alpha n+1-\alpha)}, 0<\alpha<1 .
$$

where $\Gamma$ is a Gamma function.

It is known that $M_{\alpha}(z)$ satisfies the following equality (see [12, (F.333)])

$\int_{0}^{\infty} r^{\delta} M_{\alpha}(r) d r=\frac{\Gamma(\delta+1)}{\Gamma(\alpha \delta+1)}, \delta>-1,0<\alpha<1$.

\section{MAIN RESUltS}

In this section we prove the existence of a mild solution of (1.1) by using the Hausdorff measure of noncompactness. The function $g$ is assumed to be compact.

A function $u \in C([0, b] ; X)$ is called a mild solution of the equation (1.1) if

$u(t)=T_{\alpha}(t) g(u)+\int_{0}^{t}(t-s)^{\alpha-1} S_{\alpha}(t-s) f(s, u(\sigma(s))) d s$,

where $T_{\alpha}(t)=\int_{0}^{\infty} M_{\alpha}(r) T\left(t^{\alpha} r\right) d r, t \geq 0$,

$S_{\alpha}(t)=\int_{0}^{\infty} \alpha r M_{\alpha}(r) T\left(t^{\alpha} r\right) d r, t \geq 0$,

where $M_{\alpha}(r)$ is the Mainardi's function.

\subsection{Remark}

If $\mathrm{T}(\mathrm{t})$ is equicontinuous, by (2.2), it is easy to show that $T_{\alpha}(t), S_{\alpha}(t)$ are equicontinuous.

\subsection{Lemma}

Let $0<\alpha<1$. Let the semigroup $T(t)$ be equicontinuous and $\varphi \in L^{1}\left([0, b] ; \mathbb{R}_{+}\right)$. Then the set $\left\{\int_{0}^{t}(t-s)^{\alpha-1} S_{\alpha}(t-s) u(s) d s,\|u(s)\| \leq \varphi(s)\right.$ a.e. $\left.s \in[0, b]\right\}$ is equicontinuous for $t \in[0, b]$. 
Proof: For $0 \leq t_{1} \leq t_{2} \leq b$, we have

$$
\begin{aligned}
& \left\|\int_{0}^{t_{2}}\left(t_{2}-s\right)^{\alpha-1} S_{\alpha}\left(t_{2}-s\right) u(s) d s-\int_{0}^{t_{1}}\left(t_{1}-s\right)^{\alpha-1} S_{\alpha}\left(t_{1}-s\right) u(s) d s\right\| \\
& =\| \int_{0}^{t_{1}}\left(t_{2}-s\right)^{\alpha-1} S_{\alpha}\left(t_{2}-s\right) u(s) d s-\int_{0}^{t_{1}}\left(t_{1}-s\right)^{\alpha-1} S_{\alpha}\left(t_{2}-s\right) u(s) d s \\
& +\int_{t_{1}}^{t_{2}}\left(t_{2}-s\right)^{\alpha-1} S_{\alpha}\left(t_{2}-s\right) u(s) d s+\int_{0}^{t_{1}}\left(t_{1}-s\right)^{\alpha-1} S_{\alpha}\left(t_{2}-s\right) u(s) d s \\
& -\int_{0}^{t_{1}}\left(t_{1}-s\right)^{\alpha-1} S_{\alpha}\left(t_{1}-s\right) u(s) d s \| \\
& \leq\left\|\int_{0}^{t_{1}}\left(\left(t_{2}-s\right)^{\alpha-1}-\left(t_{1}-s\right)^{\alpha-1}\right) S_{\alpha}\left(t_{2}-s\right) u(s) d s\right\| \\
& +\left\|\int_{0}^{t_{1}}\left(t_{1}-s\right)^{\alpha-1}\left(S_{\alpha}\left(t_{1}-s\right)-S_{\alpha}\left(t_{2}-s\right)\right) u(s) d s\right\| \\
& +\left\|\int_{t_{1}}^{t_{2}}\left(t_{2}-s\right)^{\alpha-1} S_{\alpha}\left(t_{2}-s\right) u(s) d s\right\| \\
& \leq \int_{0}^{t_{1}} \|\left(\left(t_{2}-s\right)^{\alpha-1}-\left(t_{1}-s\right)^{\alpha-1}\|\| S_{\alpha}\left(t_{2}-s\right) u(s) \| d s\right. \\
& +\left\|\int_{0}^{t_{1}}\left(t_{1}-s\right)^{\alpha-1}\right\| S_{\alpha}\left(t_{2}-s\right)-S_{\alpha}\left(t_{1}-s\right)\|\| u(s)\|d s\| \\
& +\int_{t_{1}}^{t_{2}}\left(t_{2}-s\right)^{\alpha-1}\left\|S_{\alpha}\left(t_{2}-s\right) u(s)\right\| d s .
\end{aligned}
$$

From this inequality it follows that

$$
\left\|\int_{0}^{t_{2}}\left(t_{2}-s\right)^{\alpha-1} S_{\alpha}\left(t_{2}-s\right) u(s) d s-\int_{0}^{t_{1}}\left(t_{1}-s\right)^{\alpha-1} S_{\alpha}\left(t_{1}-s\right) u(s) d s\right\| \rightarrow 0
$$

as $t_{1} \rightarrow t_{2}$. The proof is complete.

\subsection{Lemma ([8]).}

Suppose $b \geq 0, \sigma>0$ and $a(t)$ is a nonnegative function locally integrable on $0 \leq t<T$ (some $T \leq+\infty)$, and suppose $c(t)$ is nonnegative and locally integrable on $0 \leq t<T$ with

$$
c(t) \leq a(t)+b \int_{0}^{t}(t-s)^{\sigma-1} c(s) d s
$$

on this interval. Then

$$
c(t) \leq a(t)+\mu \int_{0}^{t} E_{\sigma}^{\prime}(\mu(t-s)) a(s) d s, 0 \leq t<T,
$$

where $\mu=(b \Gamma(\sigma))^{1 / \sigma}$,

$$
E_{\sigma}(z)=\sum_{n=0}^{\infty} z^{n \sigma} / \Gamma(n \sigma+1),
$$

$E_{\sigma}^{\prime}(z)=\frac{d}{d z} E_{\sigma}(z)$. If $a(t) \equiv a$, constant, then $c(t) \leq a E_{\sigma}(\mu t)$.

To prove the main results, we use the following assumptions:

(A1) The $C_{0}$ semigroup $T(t)$ is equicontinous, and there exists a constant $M \geq 1$ such that 
$\sup _{t \geq 0}\|T(t)\| \leq M$.

(3.4)

(A2) $g: C([0, b] ; X) \rightarrow X$ is continuous and compact, and there exists and a constant $N>0$ such that $\|g(u)\| \leq N, u \in C([0, b] ; X)$.

(A3) $f:[0, b] \times X \rightarrow X$ satisfied the Caratheodory condition; i.e. $f(\cdot, x)$ is measurable for all $x \in X$, and $f(t, \cdot)$ is continuous for a.e. $t \in[0, b]$.

(A4) $\sigma:[0, b] \rightarrow[0, b]$ is continuous function such that $\sigma(t) \leq t, t \in[0, b]$

(A5) There exists a function $h:[0, b] \times \mathbb{R}_{+} \rightarrow \mathbb{R}_{+}$such that $h(\cdot, s) \in L^{1}\left([0, b] ; \mathbb{R}_{+}\right)$for all $s \geq 0$,

$h(t, \cdot)$ is continuous and increasing for a.e.t $\in[0, b]$, and $\|f(t, x)\| \leq h(t,\|x\|)$ for a.e. $t \in[0, b]$

and all $x \in X$. moreover, there exists at least one solution to the following scalar equation:

$q(t)=M N+\frac{\alpha M}{\Gamma(\alpha+1)} \int_{0}^{t}(t-s)^{\alpha-1} h(s, q(s)) d s, t \in[0, b]$.

(A6) There exists a constant $\gamma>0$ such that $\beta(f(t, B(t)) \leq \gamma \beta(B(t))$.

for a.e. $t, s \in[0, b]$ and every bounded $B \subset C([0, b] ; X)$.

\subsection{Lemma}

Assume that conditions (A1) to (A6) are satisfied. Then the nonlocal problem (1.1) has at least one mild solution on $[0, \mathrm{~b}]$.

Proof: Define the mapping $F: C([0, b] ; X) \rightarrow C([0, b] ; X)$ by

$(F u)(t)=T_{\alpha}(t) g(u)+\int_{0}^{t}(t-s)^{\alpha-1} S_{\alpha}(t-s) f(s, u(\sigma(s))) d s, t \in[0, b]$.

It is obvious that the fixed point of $F$ is the mild solution of (1.1) and it is easy to show that $F$ is continuous on $C([0, b] ; X)$. From (3.2), (2.2) and (3.4), it follows that $\left\|T_{\alpha}(t)\right\| \leq M, t \geq 0$.

From(3.3) and (2.2), it follows that

$\left\|S_{\alpha}(t)\right\| \leq \alpha M \int_{0}^{\infty} r M_{\alpha}(r) d r \leq \frac{\alpha M}{\Gamma(\alpha+1)}, t \geq 0$.

Set $Q_{0}=\{u \in C([0, b] ; X),\|u(t)\| \leq q(t), t \in[0, b]\}$. Then $Q_{0} \subset C([0, b] ; X)$ is bounded and convex. Define $Q_{1}=\overline{\operatorname{conv}} F\left(Q_{0}\right)$, where $\overline{\operatorname{conv}}$ means the closure of the convex hull in $C([0, b] ; X)$.

From Remark 3.1, Lemma 3.2, (A4), the equicontinuity of $T(t)$, compactness of $g$ and $Q_{0} \subset$ $C([0, b] ; X)$, it follows that $Q_{1} \subset C([0, b] ; X)$ is bounded closed convex and equicontinuous on $[0, \mathrm{~b}]$.

For every $u \in F\left(Q_{0}\right),\|u(t)\| \leq M N+\frac{\alpha M}{\Gamma(\alpha+1)} \int_{0}^{t}(t-s)^{\alpha-1} h(s, q(s)) d s=q(t)$. This implies

$Q_{1} \subset Q_{0}$. We denote $Q_{n+1}=\overline{\operatorname{conv}} F\left(Q_{n+1}\right), n=1,2, \ldots$. It is easy to show that $\left\{Q_{n+1}\right\}_{n=1}^{\infty}$ is a decreasing sequence of equicontinuous on $[0, \mathrm{~b}]$, and is bounded closed convex subsets of $C([0, b] ; X)$. Since $X$ is separable, then $C([0, b] ; X)$ is separable, hence there exists a dense subset $\left\{u_{k}\right\}_{n=1}^{\infty}$ of $Q_{n}$. From Lemma 2.2, it follows that

$$
\begin{gathered}
\beta\left(Q_{n+1}(t)\right)=\beta\left(\cup_{k=1}^{\infty}\left(F u_{k}\right)(t)\right) \\
\leq \beta\left(\left\{T_{\alpha}(t) g\left(\cup_{k=1}^{\infty} u_{k}\right)+\cup_{k=1}^{\infty} \int_{0}^{t}(t-s)^{\alpha-1} S_{\alpha}(t-s) f\left(s, u_{k}(\sigma(s))\right) d s\right\}\right)
\end{gathered}
$$

Since $g$ is compact, by Lemma 2.5, we have

$$
\begin{gathered}
\beta\left(Q_{n+1}(t)\right) \leq \beta\left(\cup_{k=1}^{\infty} \int_{0}^{t}(t-s)^{\alpha-1} S_{\alpha}(t-s) f\left(s, u_{k}(\sigma(s))\right) d s\right) \\
\leq \int_{0}^{t}(t-s)^{\alpha-1} \beta\left(\left\{S_{\alpha}(t-s) f\left(s, \cup_{k=1}^{\infty} u_{k}(\sigma(s))\right) d s\right\}\right)
\end{gathered}
$$




$$
\begin{gathered}
\leq \int_{0}^{t}(t-s)^{\alpha-1} \beta\left(\left\{S_{\alpha}(t-s) f\left(s, \cup_{k=1}^{\infty} u_{k}(s)\right) d s\right\}\right) \\
\quad \leq \int_{0}^{t}(t-s)^{\alpha-1} \beta\left(S_{\alpha}(t-s) f\left(s, Q_{n}(s)\right) d s\right) \\
\quad \leq \frac{\alpha M}{\Gamma(\alpha+1)} \int_{0}^{t}(t-s)^{\alpha-1} \beta\left(f\left(s, Q_{n}(s)\right) d s .\right.
\end{gathered}
$$

By (3.6) and (3.10), we have

$\beta\left(Q_{n+1}(t)\right) \leq \frac{\alpha \gamma M}{\Gamma(\alpha+1)} \int_{0}^{t}(t-s)^{\alpha-1} \beta\left(\left(Q_{n}(s)\right) d s\right.$.

(3.11)

Since $Q_{n}$ is decreasing with respect to $n$, we define

$\theta(t)=\lim _{n \rightarrow \infty} \beta\left(Q_{n}(t)\right), t \in[0, b]$.

Taking $n \rightarrow \infty$ to both sides of (3.11), we have

$\theta(t) \leq \frac{\alpha \gamma M}{\Gamma(\alpha+1)} \int_{0}^{t}(t-s)^{\alpha-1} \theta(s) d s$.

By Lemma 3.3, we obtain $\theta(t)=0, t \in[0, b]$. By Lemma 2.3, $\lim _{n \rightarrow \infty} \beta\left(Q_{n}\right)=0$. By Lemma 2.2, it follows that $Q=\cap_{n=1}^{\infty} Q_{n}$ is convex compact in $C([0, b] ; X)$ and $F(Q) \subset Q$. From the Schauder fixed point theorem, there exists at least one fixed point $u \in Q$, which is mild solution of (1.1).

\section{REFERENCES}

[1] J. Banas, K.Goebel, Measure of Noncompactness in Banach spaces, Lect. Notes Pure Appl. Math., vol.60, Marcel Dekker, New York, 1980.

[2] E. Bazhlekova, Fractional Evolution Equations in Banach spaces, Ph.D. Thesis, Eindohoven University of Technology, 2001.

[3] D.Bothe, Multivalued perturbations of m-accretive differential inclusions, Israel J. Math. 108, 1998, 109-138.

[4] L.Byszewski, Theorems about the existence and uniqueness of solutions of a semilinear evolution nonlocal Cauchy problem, J.Math. Anal. Appl. 162, 1991, 494-505.

[5] Z. Fan, G. Li, Existence results for semiliinear differential equations with nonlocal and impulsive conditions, J.Funct. Anal. 258, 2010, 1709-1727.

[6] X.Fu, K. Ezzinbi, Existence of solutions of a semilinear functional differential evolution equations with nonlocal conditions, Nonlinear Anal. 54, 2003, 215-227.

[7] M.Hahn, K.Kobayashi, S.Umarov, Fokker-Planck-Kolmogorov equations associated with time changed fractional Brownian motion, Proc.Amer.Math.Soc.13, 2011, 691-705.

[8] D.Henry, Geometric Theory of Semilinear Parabolic Equations, Lecture Notes in Math., vol.840, Springer-verlag, New York, Berlin, 1981.

[9] A.A.Kilbas, H.M. Srivastava, J.J.Trujillo, Theory and Applications of Fractional Differential Equations, North Holland Mathematics Studies, vol.204, Elsevier Science B.V., Amsterdam, 2006.

[10] K.Li, J.Peng, J. Gao, Nonlocal Fractional Semilinear differential equations in separable Banach spaces, Elec. J. Diff. Eq., vol.2013, no.7, 2013, 1-7.

[11] Y.Lin, J.Liu, Semilinear integrodifferential equations with nonlocal Cauchy problem, Nonlinear Anal. 26, 1996, 1023-1033.

[12] F. M.Mainardi, Functional Calculus and Waves in Linear Viscoelasticity, An introduction to Mathematical Models ( Imperial College Press, 2010.)

[13] M. M. Meerschaert, E. Nane, E. Vellaisamy, Fractional Cauchy problems on bounded domains, Ann. Probab. 37 ,2009, 979-1007.

[14] R.R. Nigmatullin, To the theoretical explanation of the "universal response". Phys. Stat. Sol. (b), 123, 1984, 739-745.

[15] S.Ntouyas, P.Tsamatos, Global existence for semilinear evolution equations with nonlocal conditions, J. Math. Anal. Appl. 210 1997, 679-687. 
[16] E. Orsingher, L. Beghin, Time-fractional telegraph equations and telegraph processes with Brownian time, Probab. Theory. Related. Fields. 128, 2004, 141-160.

[17] I. Podulbny, Fractional Differential Equations, (Academic Press, New York 1999).

[18] S.G. Samko, A.A. Kilbas, O.I. Marichev, Fractional Integrals and Derivatives, Theory and Applications, Gordon and Breach, Yverdon, 1993.

[19] X.Xue, Semilinear nonlocal differential equations with measure of noncompactness in Banach spaces, J. Nanjing. Univ. Math. 24 , 2007, 2164-276.

[20] X.Xue, Existence of semilinear differential equations with nonlocal initial conditions, Acta. Math, Sini., 23, 2007, 983-988. 